\title{
原子层沉积工艺制备催化薄膜厚度对生长碳纳米管阵列的影响
}

\author{
杨超, 李莹, 间璐, 曹暳真 \\ (中国科学院 上海硅酸盐研究所, 特种无机涂层重点实验室, 上海 200050)
}

\begin{abstract}
摘 要: 通过原子层沉积(ALD)工艺在硅基底依次沉积氧化铝缓冲层薄膜和氧化铁催化薄膜, 然后利用管式炉进行 水辅助化学气相沉积(WACVD)生长垂直碳纳米管阵列(VACNTs)。结果表明: ALD 工艺制备的氧化铁薄膜经还原气 氛热处理可形成碳纳米管阵列生长所需的纳米催化颗粒; 氧化铁薄膜厚度与纳米催化颗粒大小以及生长出的碳纳 米管阵列的结构密切相关。当氧化铁薄膜厚度为 $1.2 \mathrm{~nm}$ 时, 生长出的碳纳米管阵列管外径约为 $10 \mathrm{~nm}$, 管壁层数约 为 5 层, 阵列高度约为 $400 \mu \mathrm{m}$ 。增大氧化铁薄膜的厚度, 生长出的碳纳米管阵列外径和管壁数增加, 阵列高度降低。 实验还在硅基底侧面观察到了 VACNTs, 表明 ALD 工艺可在三维结构上制备催化薄膜用于生长 VACNTs。

关 键 词: 原子层沉积; 氧化铁; 水辅助化学气相沉积; 垂直碳纳米管阵列; 结构可控; 三维样品

中图分类号: TQ174 文献标识码: A
\end{abstract}

\section{Influence of Catalyst Film Thickness Deposited by Atomic Layer Deposition on Growth of Aligned Carbon Nanotubes}

\author{
YANG Chao, LI Ying, YAN Lu, CAO Yun-Zhen
}

(Key Laboratory of Inorganic Coating Materials, Shanghai Institute of Ceramics, Chinese Academy of Sciences, Shanghai 200050, China)

\begin{abstract}
Al}_{2} \mathrm{O}_{3}$ buffer layer and $\mathrm{Fe}_{2} \mathrm{O}_{3}$ catalyst film were deposited on $\mathrm{Si}$ wafer successively by atomic layer deposition (ALD). The coated Si wafer was used to grow vertically aligned carbon nanotubes (VACNTs) by water-assisted chemical vapor deposition (WACVD). Results show that catalytically active nanoparticles form in the ALD deposited $\mathrm{Fe}_{2} \mathrm{O}_{3}$ film after heat-treatment in reduced atmosphere, and the thickness of $\mathrm{Fe}_{2} \mathrm{O}_{3}$ film is closely related to the size of catalytic nanoparticles and the structure of VACNTs grown. For 1.2 nm-thick $\mathrm{Fe}_{2} \mathrm{O}_{3}$ film as catalyst layer, the VACNTs have an outer diameter of $\sim 10 \mathrm{~nm}$, wall numbers of $\sim 5$ and height of $\sim 400 \mu \mathrm{m}$. Increasing the thickness of $\mathrm{Fe}_{2} \mathrm{O}_{3}$ film leads to outer diameter and wall number of VACNTs increasing, but the height of which decreasing. It is also observed that VACNTs grows on the side face of Si wafer, which indicates that ALD technique can be useful for VACNTs growth on three-dimensional substrate.
\end{abstract}

Key words: atomic layer deposition; iron oxide; water-assisted chemical vapor deposition; vertically aligned carbon nanotubes; controlled structure; three-dimensional sample

垂直碳纳米管阵列 (VACNTs) 是碳纳米管在范 德瓦尔斯力的作用下形成的紧密排列的宏观结构 ${ }^{[1]}$, 它特有的纳米结构和性能使其在超黑材料 ${ }^{[2]}$ 、传感 $\mathrm{g}^{\mathrm{g}}{ }^{[3]}$ 、超级电容器电极 ${ }^{[4]}$ 和纳滤膜 ${ }^{[5]}$ 等方面有着广泛 的应用。采用化学气相沉积法(CVD)生长碳纳米管
时, 需先在基底表面沉积缓冲层薄膜和催化剂薄 膜。常用的缓冲层为 $\mathrm{Al}_{2} \mathrm{O}_{3}$ 薄膜 ${ }^{[6]}$, 其作用是在高温 条件下帮助催化薄膜形成纳米颗粒并阻止基底与催 化剂发生化学反应。而 Fe 或 Fe 的氧化物因活性高、 价格低廉成为最常用的催化剂 ${ }^{[7]}$ 。在 CVD 生长碳 
纳米管阵列过程中, 催化剂薄膜在还原气氛下经 $750{ }^{\circ} \mathrm{C}$ 左右的热处理, 在表面张力和奥斯瓦尔德成 熟机制的共同作用下形成纳米催化颗粒。碳源分子 在催化剂纳米颗粒作用下裂解产生碳原子, 经过表 面吸附、扩散、溶解、重排并达到饱和后, 析出形 成碳纳米管 ${ }^{[8]}$ 。研究表明在反应过程中引入水蒸气 可起到保持催化剂的活性 ${ }^{[9]}$ 和消除无定型碳 ${ }^{[10]}$ 的作 用, 从而促进 VACNTs 的生长。其中, 铁纳米颗粒 作为碳纳米管成核生长的位点, 对碳纳米管的管壁 数量、直径大小等起着决定性的作用 ${ }^{[11]}$, 但用于生 长 VACNTs 的铁或氧化铁催化薄膜较薄(1 5 nm), 其厚度的细微变化, 对形成的铁纳米颗粒的形貌都 有较大影响, 因此制备均匀的 1 5 nm 厚的铁或氧 化铁薄膜就显得尤为重要。另外, 考虑到 VACNTs 在某些复杂三维结构上的应用, 还需要在复杂三维 结构上制备催化薄膜。

原子层沉积工艺是一种基于表面饱和吸附自限 制反应的成膜方法, 能够在复杂三维结构上均匀成 膜 ${ }^{[12]}$ 。目前, 关于 ALD 工艺制备 VACNTs 生长所 需的催化薄膜的文献报道较少。ZHOU 等 ${ }^{[13]}$ 利用 ALD 工艺制备氧化铁催化剂, 但采用电子束蒸镀工 艺制备氧化铝缓冲层; PLACIDUS 等 ${ }^{[14]}$ 利用 ALD工 艺制备氧化铝缓冲层, 但采用电子束蒸镀工艺制备 氧化铁催化剂; CHEN 等 ${ }^{[15]}$ 利用 ALD 工艺同时制备 了氧化铝缓冲层和氧化铁催化剂组成的催化薄膜, 并催化生长出 VACNTs, 但是文中没有深入讨论 ALD 氧化铁薄膜的厚度与 VACNTs 结构的关系。

本工作采用 ALD 工艺, 使用氯化铁和水作为 前驱体, 在 ALD 氧化铝表面成功制备出不同厚度 的氧化铁薄膜, 采用 WACVD 工艺生长出 VACNTs, 并研究了 ALD 氧化铁薄膜厚度与 VACNTs 的管径 大小、管壁层数、纯度以及阵列高度的关系。

\section{1 实验方法}

\section{1 氧化铝缓冲层和氧化铁催化剂的制备}

实验所用祄底为 $\mathrm{P}(110)$ 单面抛光硅片, 厚度约 为 $725 \mu \mathrm{m}$, 采用乙醇、丙醞、去离子水依次超声清 洗, 然后烘干备用。采用芬兰 BENEQ-TFS500 原子 层沉积系统制备氧化铝和氧化铁薄膜, 反应前驱体 和吹扫气体 $\mathrm{Ar}$ 通过脉冲的方式进入反应室, 过程如 下: (1)第一种前驱体进入反应室, 时间为 $t_{1}$; (2)通 入 $\mathrm{Ar}$, 时间为 $t_{2}$; (3)通入第二种前驱体, 时间为 $t_{3}$; (4)通入 $\mathrm{Ar}$, 时间为 $t_{4}$ 。如此为一个循环, 通过调整 循环次数来控制薄膜的厚度, 实验控制氧化铝薄
膜的厚度为 $12 \mathrm{~nm}$, 氧化铁薄膜的厚度为 $1.2 、 2.4$ 、 $4.8 \mathrm{~nm}$ 。

氧化铝和氧化铁的反应室温度为 $225^{\circ} \mathrm{C}$, 腔室 真空度为 $100 \mathrm{~Pa}$ 。实验中沉积氧化铝的前驱体为三 甲基铝和水，反应方程为:

$$
\begin{aligned}
\mathrm{AlOH}^{*}+\mathrm{Al}\left(\mathrm{CH}_{3}\right)_{3} & \rightarrow \mathrm{AlOAl}\left(\mathrm{CH}_{3}\right)_{2} *+\mathrm{CH}_{4} \\
\mathrm{AlCH}_{3} *+\mathrm{H}_{2} \mathrm{O} & \rightarrow \mathrm{AlOH}^{*}+\mathrm{CH}_{4}
\end{aligned}
$$

沉积氧化铁的前驱体为三氯化铁和水, 反应方程为:

$$
x \mathrm{OH}^{*}+\mathrm{Fe}_{2} \mathrm{Cl}_{6} \rightarrow \mathrm{Fe}_{2} \mathrm{O}_{x} \mathrm{Cl}_{6-x} *+x \mathrm{HCl}
$$

$\mathrm{Fe}_{2} \mathrm{O}_{x} \mathrm{Cl}_{6-x} *+3 \mathrm{H}_{2} \mathrm{O} \rightarrow \mathrm{Fe}_{2} \mathrm{O}_{3}(\mathrm{OH})_{x} *+(6-x) \mathrm{HCl}$ (4)

\subsection{VACNTs 的生长}

采用 WACVD 工艺制备 VACNTs, 设备采用合 肥科晶的 OTF-1200X 真空管式炉。 $\mathrm{Ar}$ 经过 $0^{\circ} \mathrm{C}$ 恒温 的去离子水槽携带水蒸气进入管式炉, 标注为 $\operatorname{Ar}\left(\mathrm{H}_{2} \mathrm{O}\right)$ 。制备过程如下: (1)将样品置于管式炉中, 抽真空(600 Pa); (2)通入 400sccm Ar 作为保护气, 加 热到 $750^{\circ} \mathrm{C}$; (3)保持 $\mathrm{Ar}$ 流量不变, 通入 $100 \mathrm{sccm} \mathrm{H}$ 进行氧化铁的还原, $5 \mathrm{~min}$ 后关闭 $\mathrm{H}_{2}$; (4)通入 $900 \mathrm{sccm} \mathrm{Ar}, 70 \mathrm{sccm} \mathrm{C}_{2} \mathrm{H}_{2}, 30 \mathrm{sccm} \operatorname{Ar}\left(\mathrm{H}_{2} \mathrm{O}\right)$ 生长 VACNTs10 min; (5)通入 Ar100sccm 作为保护气, 其 他气体阀门关闭, 自然冷却到室温, 关闭真空愿恢 复常压, 取出样品。

\section{3 材料表征}

采用 ESCALAB 250 型 X 射线光电子能谱仪(XPS) 表征氧化铁中铁的价态。采用 BRUKER Dimension Icon 原子力显微镜对催化剂表面形貌进行表征, 测 量模式为 ScanAsyst, 扫描范围为 $500 \mathrm{~nm} \times 500 \mathrm{~nm}$, 分析软件为 Nanoscope Analysis 1.5。采用 HITACHI S-3400N 铇灯丝扫描电镜(T-SEM)观察 VACNTs 的 形貌, 测试电压为 $15 \mathrm{kV}$, 电流 $0.75 \mathrm{~mA}$ 。采用高分 辨率 SU8220 场发射扫描电子显微镜(F-SEM) 观察 VACNTs 的内部形貌, 测试电压为 $3 \mathrm{kV}$, 电流 $0.1 \mathrm{~mA}$ 。 采用 F20 场发射透射电子显微镜(TEM)观察碳纳米 管的管径和管壁。采用 Horiba Lab-Ram iHR550 表 征 VACNTs 的拉曼信息, 测量激光波长为 $632 \mathrm{~nm}$, 测量波数范围为 $400 \sim 3000 \mathrm{~cm}^{-1}$, 步幅 $10 \mathrm{~cm}^{-1} / \mathrm{s}$ 。

\section{2 结果与讨论}

\section{1 催化薄膜的制备与分析}

在 ALD 沉积薄膜的过程中, 反应前驱体和吹 扫气体 $\mathrm{Ar}$ 通过脉冲方式进入反应室, 过程如下: (1) 第一种前驱体进入反应室, 时间为 $t_{1}$, 在基底发生 化学吸附; (2)通入 $\mathrm{Ar}$, 时间为 $t_{2}$, 吹走没有吸附的 多余前驱体; (3)通入第二种前驱体，时间为 $t_{3}$, 与第 
一种前驱体反应; (4)通入 $\mathrm{Ar}$, 时间为 $t_{4}$, 吹走没有 反应的多余第二种前驱体和没有吸附的反应物。如 此为一个循环, 并通过这种循环化学反应在基材表 面成膜。图 1(a)为氧化铁沉积速率与氯化铁、水、 氩气的脉冲时间的关系。其中, 薄膜的沉积厚度和 速率采用石英晶体微天平 $(\mathrm{QCM})$ 来进行实时监测。 从图 1(a)可以看出两点: (1) 以前驱体 $\left(\mathrm{FeCl}_{3}\right.$ 和 $\left.\mathrm{H}_{2} \mathrm{O}\right)$ 脉冲时间作为单一变量时, 随着脉冲时间的的延长, 氧化铁薄膜的生长速率由刚开始的快速增长到趋于 稳定, 最终生长速率稳定在约 $0.06 \mathrm{~nm} /$ cycle, 这是 由于随着前驱体脉冲时间的延长, 前驱体在样品表 面的吸附量逐渐增加直至饱和; (2)以 Ar 吹扫时间作 为单一变量时, 随着清洗时间的延长, 氧化铝薄膜 的生长速率逐渐降低并趋于稳定, 最终生长速率稳 定在约 $0.06 \mathrm{~nm} / \mathrm{cycle}$, 这是由于 $\mathrm{Ar}$ 吹扫时间不充分 时, 反应室内的前驱体在基片上方发生 CVD 化学 反应, 反应产物沉积在基片上, 使得薄膜厚度增大, 但随着 Ar 清洗时间的延长, 反应室中多余的前驱 体和其他反应产物被完全吹扫干净, 所以氧化铝薄 膜的生长速率逐渐降低并趋于稳定。通过图 1(a)的

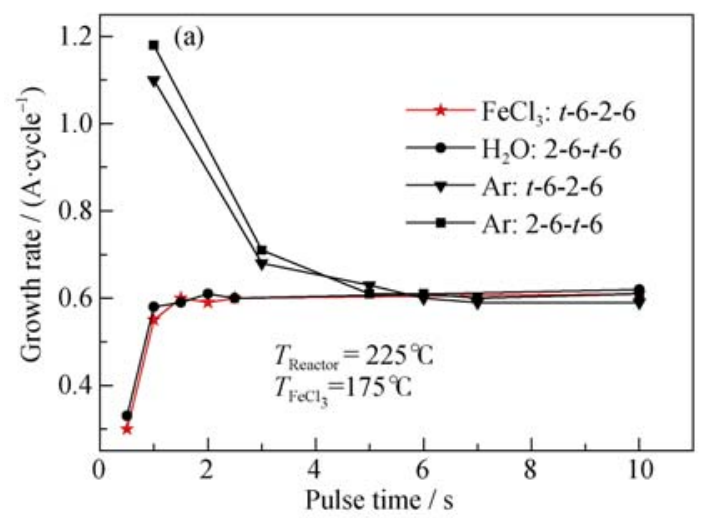

实验结果，我们选择氧化铁的循环时间为 2s-6s2s-6s，保证了薄膜的稳定沉积。采用上述相同方法， 实验获得氧化铝的循环时间为 $0.5 \mathrm{~s}-6 \mathrm{~s}-0.5 \mathrm{~s}-6 \mathrm{~s}$, 生长 速率约为 $0.12 \mathrm{~nm} /$ cycle。通过 QCM 的实时监测, 在 0 200 个循环中, 如图 1(b)所示, 氧化铝和氧化铁薄 膜的厚度随着循环次数呈线性正相关, 说明在此沉 积工艺条件下制备的氧化铝-氧化铁薄膜具有厚度 稳定可控性。

图 1(c)为 ALD 氧化铁薄膜的 Fe2p XPS 图谱, 从图中可以看出, 在结合能为 724.6、711.0 eV 处出 现两个主峰, 且峰形对称; 在结合能为 $718.8 \mathrm{eV}$ 处 出现一个较弱的峰。通过分析可知, $724.6 \mathrm{eV}$ 处峰为 $\mathrm{Fe} 2 \mathrm{p}_{1 / 2}$ 峰, $711.0 \mathrm{eV}$ 处峰为 $\mathrm{Fe} 2 \mathrm{p}_{3 / 2}$ 峰, $718.8 \mathrm{eV}$ 处峰 为氧化铁特有的 $\mathrm{Fe} 2 \mathrm{p}_{3 / 2}$ 卫星峰 ${ }^{[16]}$ 。实验结果显示, 上述 ALD 工艺在 ALD 氧化铝表面成功制备了 $\mathrm{Fe}_{2} \mathrm{O}_{3}$ 薄膜。

\section{2 催化剂厚度与 VACNTs 结构的关系}

图 2(a c) 分别为 $1.2 、 2.4 、 4.8 \mathrm{~nm}$ 厚 ALD 氧化 铁薄膜还原 $5 \mathrm{~min}$ 后的表面 AFM 照片，从图中可以 看出，随着氧化铁薄膜厚度的增加，它在基底表面
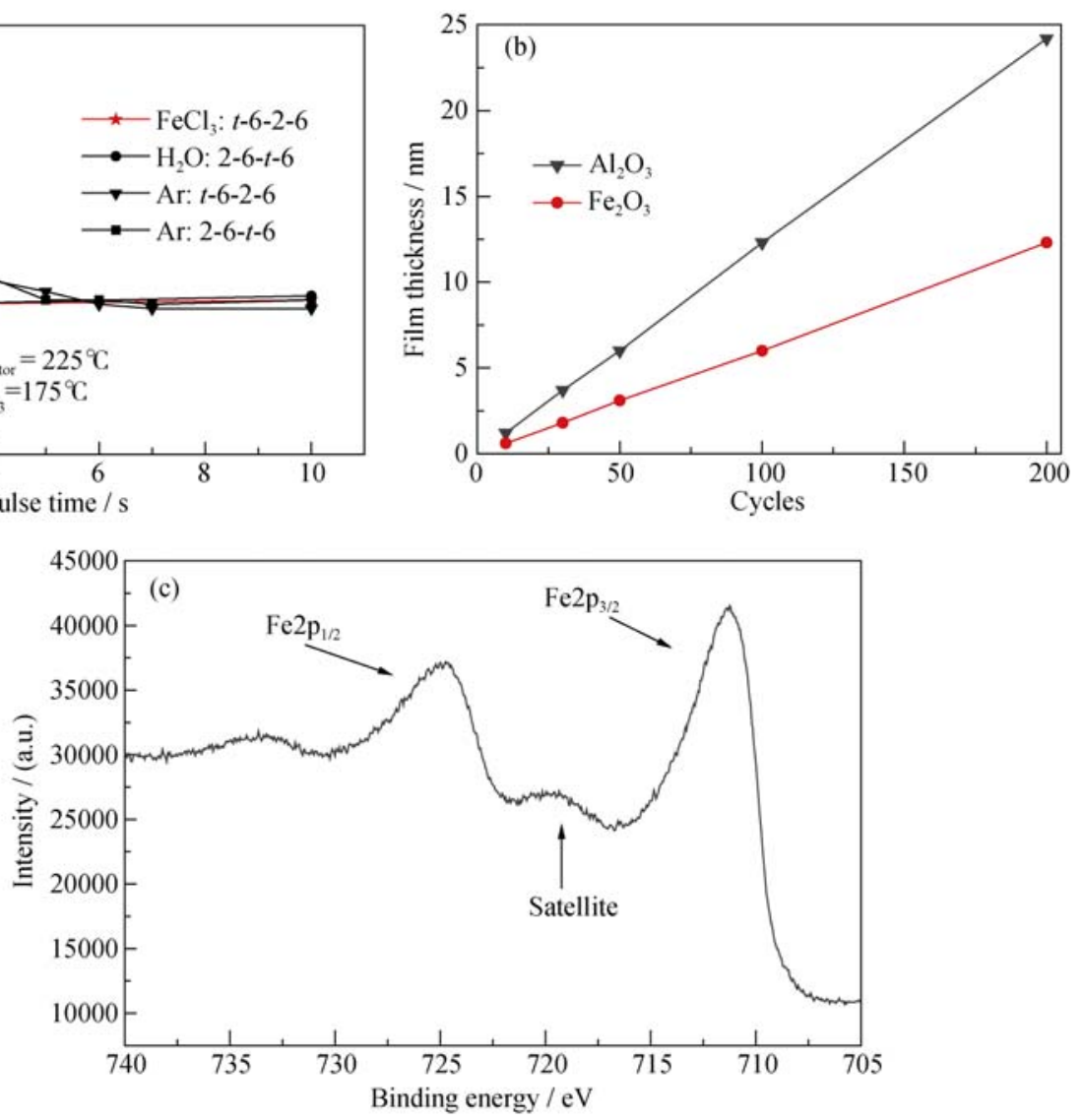

图 1 氧化铁催化薄膜的分析与表征

Fig. 1 Analysis and characterization of $\mathrm{Fe}_{2} \mathrm{O}_{3}$ for catalytic film (a) Growth rate of $\mathrm{Fe}_{2} \mathrm{O}_{3}$ versus pulse time; (b) film thickness of $\mathrm{Al}_{2} \mathrm{O}_{3}$ and $\mathrm{Fe}_{2} \mathrm{O}_{3}$ versus cycles;

(c) XPS spectrum of $\mathrm{Fe} 2 \mathrm{p}$ from $\mathrm{Fe}_{2} \mathrm{O}_{3}$ film 

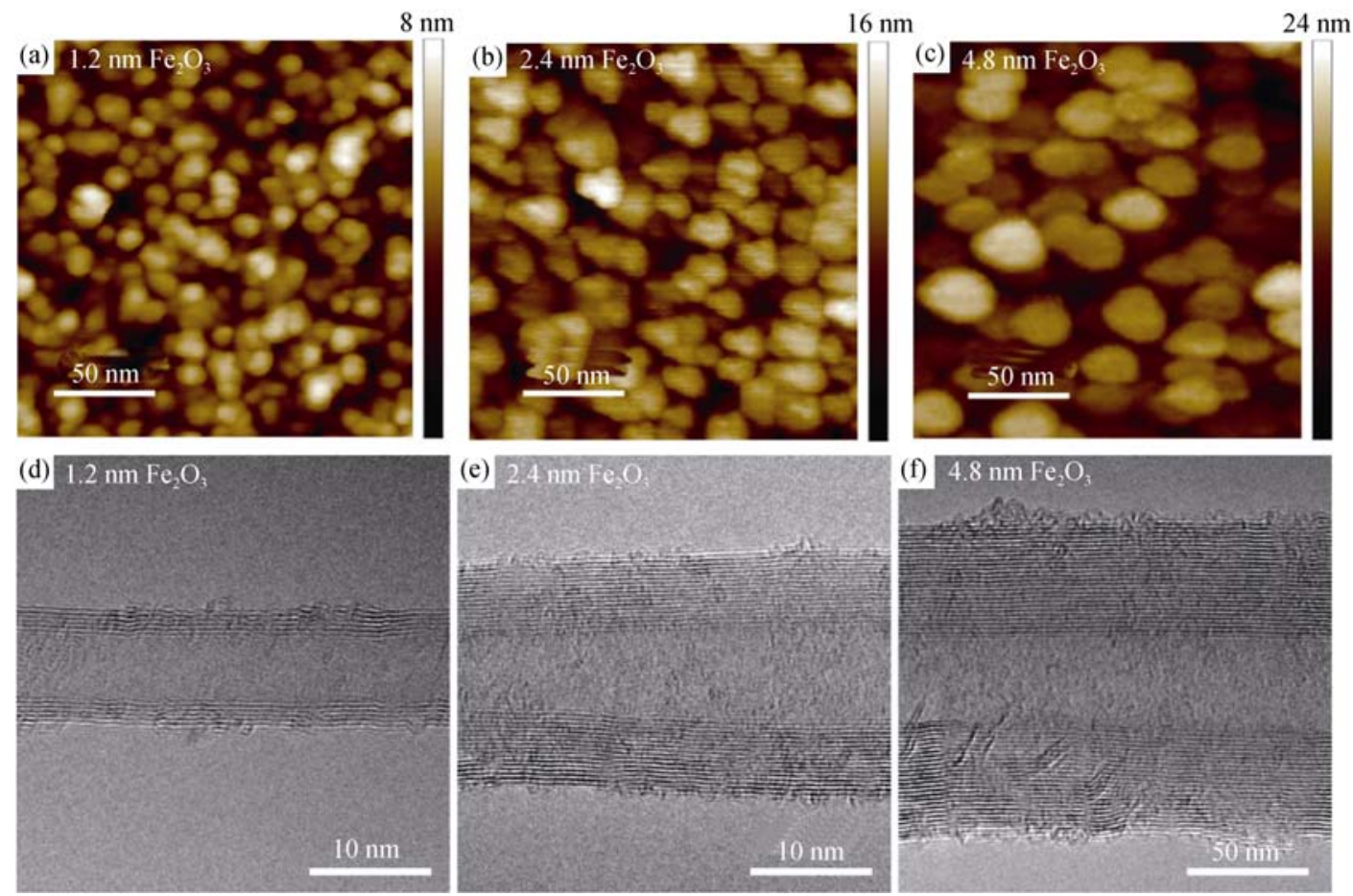

图 $2(\mathrm{a} \sim \mathrm{c})$ 不同厚度氧化铁薄膜还原 $5 \mathrm{~min}$ 后的表面 $\mathrm{AFM}$ 照片; $(\mathrm{d} \sim \mathrm{f})$ 在不同厚度氧化铁生长 CNT 的 TEM 照片

Fig. 2 Surface AFM images of $\mathrm{Fe}_{2} \mathrm{O}_{3}$ films with different thicknesses after reduced for 5 min (a-c) and TEM images of CNT grown from $\mathrm{Fe}_{2} \mathrm{O}_{3}$ films with different thicknesses (d-f)

形成的纳米颗粒的粒径逐渐增大。图 $2(\mathrm{~d} \sim \mathrm{f})$ 分别为 $1.2 、 2.4 、 4.8 \mathrm{~nm}$ 厚 ALD 氧化铁薄膜生长的单根碳 纳米管的高倍 TEM 照片, 从图中可以看出, 随着氧 化铁薄膜厚度的增加, 碳纳米管的管壁层数和管径 逐渐增大。

实验以 50 次数据采集为基数, 对数据做统计分 析。图 3(A)为不同厚度氧化铁薄膜还原 $5 \mathrm{~min}$ 后形 成纳米颗粒的粒径分布, 从图中可以看出 1.2、2.4、 $4.8 \mathrm{~nm}$ 厚氧化铁薄膜还原 $5 \mathrm{~min}$ 后所形成的颗粒粒 径分别集中在 6、18、26 nm。随着氧化铁薄膜厚度 的增大, 颗粒粒径明显增大且分布较分散, 说明厚 度较大的氧化铁薄膜还原后形成的纳米颗粒的粒径 较大且分布较分散。图 3(B)为不同厚度氧化铁薄膜 生长 VACNTs 的管外径大小分布, 从图中可以看出, 1.2、2.4、4.8 nm 厚氧化铁薄膜生长的 VACNTs 的 管外径大小分别集中在 10、20、26 nm。随着氧化 铁薄膜厚度的增大, VACNTs 的管外径明显增大且 分布较分散, 说明厚度较大的氧化铁催化生长的 VACNTs 具有较大的管外径且管径分布较分散。图 3(C)为不同厚度氧化铁生长 VACNTs 的管壁层数分 布, 从图中可以看出, 1.2、2.4、4.8 nm 氧化铁生长 的 VACNTs 的管壁层数分别集中在 5 层、16 层、35 层。随着氧化铁薄膜厚度的增大, VACNTs 的管壁层 数明显增多且分布较分散, 说明厚度较大的氧化铁 催化生长的 VACNTs 具有较多的管壁层数且管壁层
数较分散。图 3(D)为不同厚度氧化铁薄膜生长 VACNTs 的拉曼光谱, 从图中可以看出 VACNTs 两 个主峰: D 峰(约 $1325 \mathrm{~cm}^{-1}$ ) 和 $\mathrm{G}$ 峰(约 $1580 \mathrm{~cm}^{-1}$ )。D、 $\mathrm{G}$ 峰分别代表石墨烯结构的杂化缺陷和 $\mathrm{sp}^{2}$ 杂化, 因 此 $I_{\mathrm{G}} / I_{\mathrm{D}}$ 可用来表示 VACNTs 的石墨烯化的程度, 其 比值越大, 说明石墨化越高 ${ }^{[13]}$ 。通过分析, 1.2、2.4、 $4.8 \mathrm{~nm}$ 厚氧化铁薄膜生长的 VACNTs 的 $I_{\mathrm{G}} / I_{\mathrm{D}}$ 分别为 1.03、1.01、0.98, 说明厚度较大的氧化铁薄膜催化 生长的 VACNTs 的石墨烯化程度较低, 即纯度较 低。另外, 在相同条件下, 1.2、2.4、4.8 nm 厚氧化 铁薄膜生长的 VACNTs 阵列的高度分别为 $400 、 100$ 、 $50 \mu \mathrm{m}$, 说明厚度较小的催化剂易生长较长的 VACNTs。以上结果显示, 通过控制 ALD 氧化铁薄 膜的厚度可实现 VACNTs 结构可控的制备。

\subsection{VACNTs 的表征与分析}

图 4(a)为垂直 VACNTs 倾斜侧面 60的 T-SEM 照片, 氧化铁催化薄膜厚 $1.2 \mathrm{~nm}$, 从图中可以看出, 碳纳米管在祄底表面垂直紧密排列, 且高度达到约 $400 \mu \mathrm{m}$ 。在高分辨率 F-SEM 下观察垂直生长 VACNTs 内部的阵列, 如图 4(b)所示, 碳纳米管垂直而有序 的排列着。图 4(c)为 VACNTs 的 TEM 照片, 从图中 可以看出碳纳米管的中空管式, 周围没有无定形碳 和其他杂质出现, 说明采用 ALD 工艺制备氧化铝 缓冲层和氧化铁催化薄膜可以有效催化生长高纯度 VACNTs。 

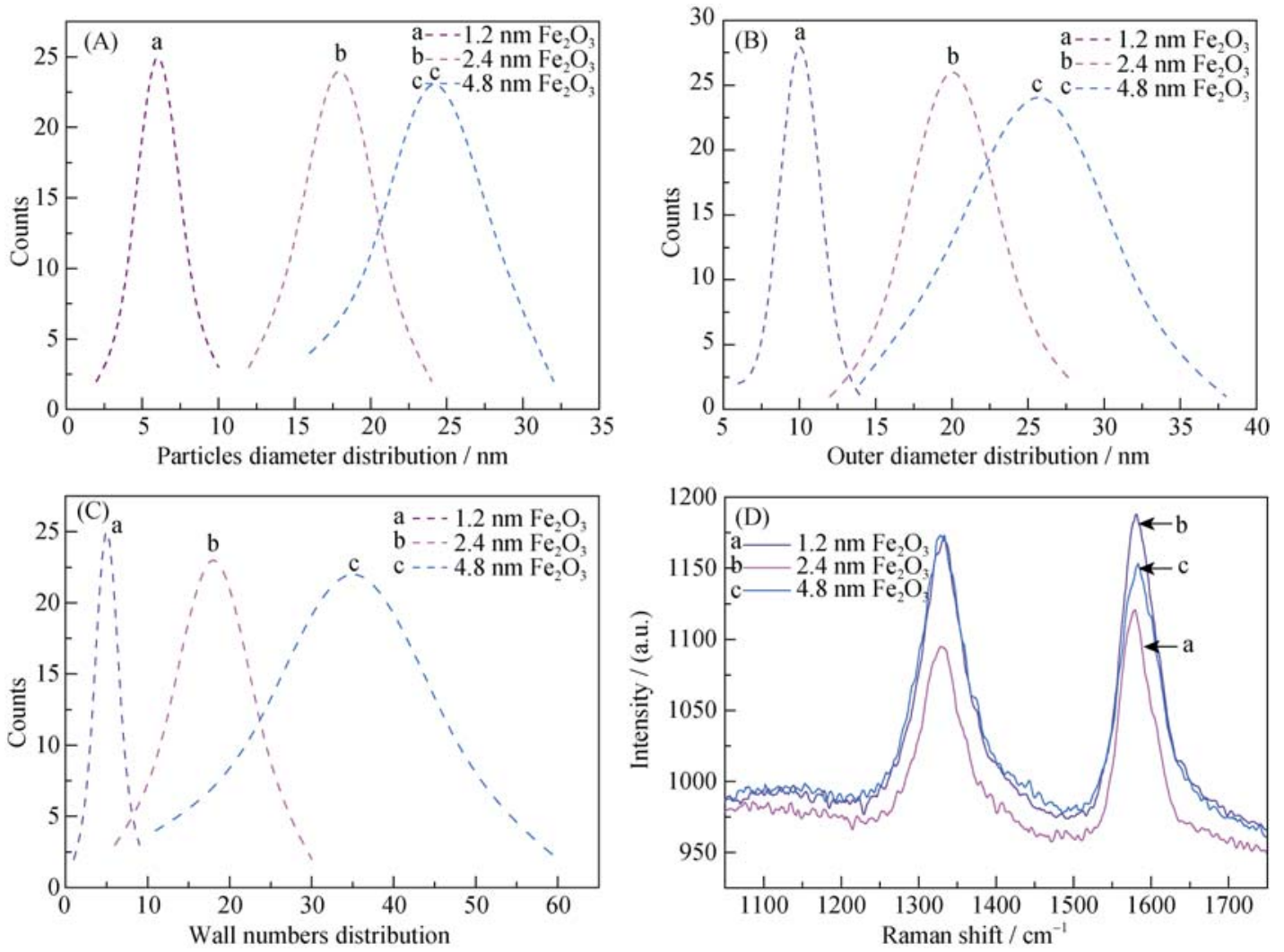

图 3 (a) 不同厚度氧化铁薄膜还原 $5 \mathrm{~min}$ 后的颗粒粒径; 不同厚度氧化铁薄膜生长 VACNTs 的(b)管外径大小、 (c)管壁层数统计 和(d)拉曼光谱分析

Fig. 3 Characterizations of $\mathrm{Fe}_{2} \mathrm{O}_{3}$ films with different thicknesses before and after being grown VACNTS

(a) Particles diameter distribution of $\mathrm{Fe}_{2} \mathrm{O}_{3}$ films with different thicknesses after reduced for 5 min; (b) Outer diameter; (c) Wall numbers distribution; (d) Raman spectra of VACNTs grown on $\mathrm{Fe}_{2} \mathrm{O}_{3}$ films with different thicknesses

实验还发现在 $725 \mu \mathrm{m}$ 厚硅片的侧面也有 VACNTs 生长, 如图 4(d) 所示。这说明原子层沉积在硅片侧边 也沉积了催化薄膜, 并能在相同 WACVD 条件下生长 垂直碳纳米管阵列, 说明 ALD 可以在三维结构上制
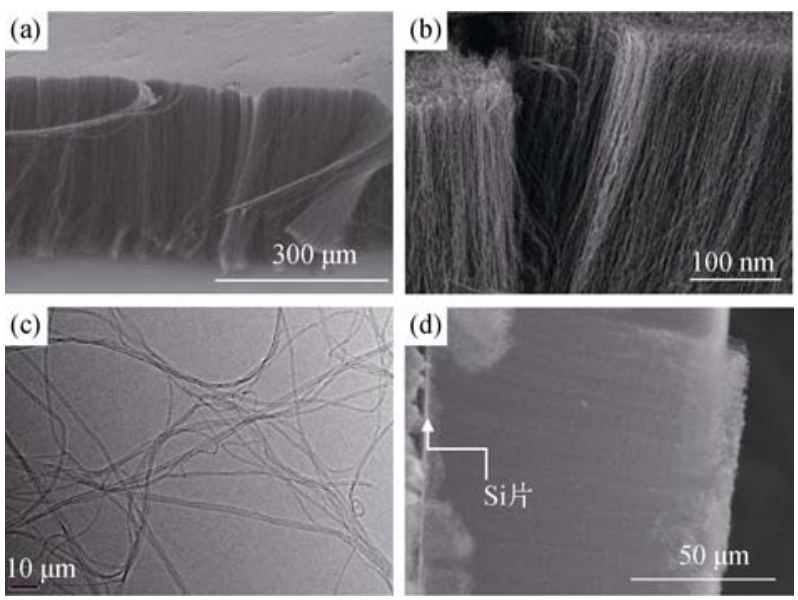

图 4 VACNTs 的 SEM 照片

Fig. 4 Morphotogy and structure of VACNTs

(a) Side-view T-SEM image of vertically aligned VACNTs; (b) Section T-SEM image of vertically aligned VACNTs; (c) TEM image of VACNTs; (d) T-SEM image of horizontally aligned VACNTs on the side of Si wafer
备催化薄膜用于生长 VACNTs。

\section{3 结论}

以氯化铁和水作为前驱体，采用 ALD 工艺在 ALD 氧化铝表面成功制备出不同厚度的氧化铁薄 膜, 并利用 WACVD 工艺生长出 VACNTs。研究了 ALD 氧化铁薄膜厚度与 VACNTs 的管径大小、管 壁层数、纯度以及阵列高度的关系: 当氧化铁薄膜 厚度为 $1.2 \mathrm{~nm}$ 时, 其管外径约为 $10 \mathrm{~nm}$, 管壁层数 约为 5 层, $I_{\mathrm{G}} / I_{\mathrm{D}}$ 为 1.03 , 阵列高度约为 $400 \mu \mathrm{m}$; 当 氧化铁薄膜厚度为 $2.4 \mathrm{~nm}$ 时, 其管外径约为 $20 \mathrm{~nm}$, 管壁层数约为 16 层, $I_{\mathrm{G}} / I_{\mathrm{D}}$ 为 1.01 , 阵列高度约为 $100 \mu \mathrm{m}$; 当氧化铁薄膜厚度为 $4.8 \mathrm{~nm}$ 时, 其管外 径约为 $26 \mathrm{~nm}$, 管壁层数约为 35 层, $I_{\mathrm{G}} / I_{\mathrm{D}}$ 为 0.98 , 阵列高度约为 $50 \mu \mathrm{m}$ 。实验结果表明, 通过调整 ALD 氧化铁薄膜的厚度可实现 VACNTs 结构可控 的制备。实验还发现, 在硅片侧面也生长出 VACNTs, 说明 ALD 工艺可以在三维结构上制备 催化薄膜用于生长 VACNTs。 


\section{参考文献:}

[1] LIANG Y X, ZHAO B, JIANG C, et al. Advances in growth control of vertically-aligned carbon nanotube forests. Chemical Industry and Engineering Progress, 2014, 33(6): 1491-1497.

[2] ANUPAMA B K, JAMES B C, MICHAEL E, et al. Ultra-high optical absorption efficiency from the ultraviolet to the infrared using multi-walled carbon nanotube ensembles. Small, 2013, 9(7): 1058-1065.

[3] YAMADA T, HAYAMIZU Y, YAMAMOTO Y, et al. A stretchable carbon nanotube strain sensor for human-motion detection. Nature Nanotechnology, 2011, 6(5): 296-301.

[4] IZADI N A, YASUDA S, KOBASHI K, et al. The full potential of single-walled carbon nanotubes as durable super capacitor electrodes. Advance Materials, 2010, 22(35): 235-241.

[5] ZHANG L, ZHAO B, WANG X, et al. Gas transport in verticallyaligned carbon nanotube/parylene composite membranes. Carbon, 2014, 66: 11-17.

[6] PLACIDUS B A, GARY L P, BENJI M, et al. Catalyst-support interactions and their influence in water-assisted carbon nanotube carpet growth. Carbon, 2012, 50(7): 2396-2403.

[7] QIU J T, YANG X J, LI Q W, et al. The role of catalysts in the controlled growth of carbon nanotubes. Chemistry Online, 2010, 12: $1086-1092$.

[8] HOFMANN S, SHARMA R, DUCATI C, et al. In situ observations of catalyst dynamics during surface-bound carbon nanotube nucleation. Nano Letter, 2007, 7(3): 602-608.

[9] PLACIDUS B A, GARY L P, BENJI M, et al. Role of water in super growth of single-walled carbon nanotube carpets. Nano Letters, 2009, 9(1): 44-49.

[10] HATA K, FUTABA D N, MIZUNO K, et al. Water-assisted highly efficient synthesis of impurity-free single-walled carbon nanotubes. Science, 2004, 306(5700): 1362-1364.

[11] ZHONG G F, WARNER J H, ROBERTSON J, et al. Growth of ultrahigh density single-walled carbon nanotube forests by improved catalyst design. ACS Nano, 2012, 6(4): 2893-2903.

[12] STEVEN M G. Atomic layer deposition: an overview. Chemical Reviews, 2010, 110(1): 111-131.

[13] ZHOU K, HUANG J Q, WEI F, et al. Multi-directional growth of aligned carbon nanotubes over catalyst film prepared by atomic layer deposition. Nanoscale Research Letters, 2010, 5(10): 1555-1560.

[14] PLACIDUS B A, CARY L P, SEUNG M K, et al. Influence of alumina type on the evolution and activity of alumina-supported $\mathrm{Fe}$ catalysts in single-walled carbon nanotube carpet growth. ACS Nano, 2010, 4(2): 895-904.

[15] CHEN B, ZHANG C, ROBERTSON J, et al. Carbon nanotube forests growth using catalysts from atomic layer deposition. Journal of Applied Physics, 2012, 115(14): 144303.

[16] YAMASHITA T, HAYES P. Analysis of XPS spectra of $\mathrm{Fe}^{2+}$ and $\mathrm{Fe}^{3+}$ ions in oxide materials. Applied Surface Science, 2008, 254(8): 2441-2449. 